\title{
Fixed Point Theorems for Occasionally Weakly Compatible Maps in $G$-Metric Space
}

\author{
Saurabh Manro \\ School of Mathematics and Computer Applications, Thapar University, \\ Patiala (Punjab) \\ Email-sauravmanro@hotmail.com, sauravmanro@yahoo.com
}

\begin{abstract}
The purpose of this paper is to prove new common fixed point theorem in Symmetric $G$-metric space. While proving our result, we utilize the idea of occasionally weakly compatible maps due to Al-Thagafi and N. Shahzad. Our result substantially generalize and improve a multitude of relevant common fixed point theorems of the existing literature in $G$ - metric space.
\end{abstract}

Index Terms - Symmetric $G$-metric space, Occasionally weakly compatible maps, Fixed point

\section{INTRODUCTION}

In 1992, Dhage[1] introduced the concept of $D-$ metric space. Recently, Mustafa and Sims[2,3] shown that most of the results concerning Dhage's $D$ - metric spaces are invalid. Therefore, they introduced a improved version of the generalized metric space structure and called it as $G$-metric space. For more details on $G$ - metric spaces, one can refer to the papers [4]-[11].

Now we give basic definitions and some basic results ([2]-[11]) which are helpful for proving our main result.

In 2006, Mustafa and Sims[3] introduced the concept of $G$-metric spaces as follows:

Definition 1.1.[3] Let $X$ be a nonempty set, and let $G$ : $X \times X \times X \rightarrow R^{+}$be a function satisfying the following axioms:
(G1) $G(x, y, z)=0$ if $x=y=z$,
(G2) $0<G(x, x, y)$, for all $\mathrm{x}, \mathrm{y} \in \mathrm{X} \quad$ with $\mathrm{x} \neq \mathrm{y}$,
(G3) $G(x, x, y) \leq G(x, y, z)$, for all $x, y, z \in X$ with $z \neq y$,

(G4) $G(x, y, z)=G(x, z, y)=G(y, z, x)=\ldots$ (symmetry in all three variables) and

(G5) $G(x, y, z) \leq G(x, a, a)+G(a, y, z)$ for all $x, y, z$, $a \in X$, (rectangle inequality)

then the function $G$ is called a generalized metric, or, more specifically a $G$ - metric on $X$ and the pair $(X, G)$ is called a $\mathrm{G}$ - metric space.

If condition (G6) also satisfied then $(X, G)$ is called Symmetric G-metric space.

(G6) $G(x, y, y)=G(x, x, y)$ for all $x, y$ in $X$.

Definition 1.2.[3] Let $(X, G)$ be a $G$-metric space then for $x_{0} \in X, r>0$, the $G$-ball with centre $x_{0}$ and radius $r$ is

$B_{G}\left(x_{0}, r\right)=\left\{y \in X: G\left(x_{0}, y, y\right)<r\right\}$.

Proposition 1.1.[3] Let $(X, G)$ be a $G$-metric space then for any $x_{0} \in X, r>0$, we have,

(1) if $G\left(x_{0}, x, y\right)<r$ then $x, y \in B_{G}\left(x_{0}, r\right)$,

(2) if y $\in \mathrm{BG}(\mathrm{x} 0, \mathrm{r})$ then there exists a $\delta>0$ such that $\mathrm{BG}(\mathrm{y}, \delta) \subseteq \mathrm{BG}(\mathrm{x} 0, \mathrm{r})$. 
It follows from (2) of the above proposition that the family of all $G$-balls,

$B=\left\{B_{G}(x, r): x \in X, r>0\right\}$ is the base of a topology $\tau$ (G) on $X$, the $G$-metric topology.

Proposition 1.2.[3] Let $(X, G)$ be a $G$-metric space then for all $x_{0} \in X$ and $r>0$, we have,

$$
B_{G}\left(x_{0}, \frac{1}{3} r\right) \subseteq B_{d_{G}}\left(x_{0}, r\right) \subseteq B_{G}\left(x_{0} r\right)
$$

where $d_{G}(x, y)=G(x, y, y)+G(x, x, y)$, for all $x, y$ in $X$. Consequently, the $G$-metric topology $\tau(\mathrm{G})$ coincides with the metric topology arising from $d_{G}$. Thus, while 'isometrically' distinct, every $G$-metric space is topologically equivalent to a metric space. This allows us to readily transport many results from metric spaces into $G$-metric spaces settings.

Definition 1.3.[3] Let $(X, G)$ be a $G$-metric space, and let $\left\{x_{n}\right\}$ a sequence of points in $X$, a point ' $x$ ' in $X$ is said to be the limit of the sequence $\left\{x_{n}\right\}$ if $\lim _{m, n \rightarrow \infty} G\left(x, x_{n}\right.$, $\left.x_{m}\right)=0$, and one says that sequence $\left\{x_{n}\right\}$ is $G$ convergent to $x$.

Thus, that if $x_{n} \rightarrow x$ or

$\lim _{n \rightarrow \infty} x_{n}=x$ in a G-metric space $(X, G)$ then for each $\varepsilon>0$, there exists a positive integer $N$ such that $G\left(x, x_{n}, x_{m}\right)<\varepsilon$ for all $m, n \geq N$.

Proposition 1.3.[3] Let $(X, G)$ be a $G$ - metric space. Then the following are equivalent:

(1) $\left\{x_{n}\right\}$ is $G$-convergent to $x$,

(2) $G\left(x_{n}, x_{n}, x\right) \rightarrow 0$ as $n \rightarrow \infty$,

(3) $G\left(x_{n}, x, x\right) \rightarrow 0$ as $n \rightarrow \infty$,

(4) $G\left(x_{m}, x_{n}, x\right) \rightarrow 0$ as $m, n \rightarrow \infty$.

Definition 1.4.[3] Let $(X, G)$ be a $\mathrm{G}$ - metric space. A sequence $\left\{x_{n}\right\}$ is called $G-$ Cauchy if, for each $\varepsilon>0$ there exists a positive integer $N$ such that
$G\left(x_{n}, x_{m}, x_{l}\right)<\varepsilon$ for all $n, m, l \geq N$

i.e. if $G\left(x_{n}, x_{m}, x_{l}\right) \rightarrow 0$ as $n, m, l \rightarrow \infty$

Proposition 1.4.[3] If $(X, G)$ is a $G$ - metric space then the following are equivalent:

(1) The sequence $\left\{\mathrm{x}_{\mathrm{n}}\right\}$ is $G$ - Cauchy,

(2) for each $\varepsilon>0$, there exist a positive integer $N$ such that

$\mathrm{G}\left(\mathrm{x}_{\mathrm{n}}, \mathrm{x}_{\mathrm{m}}, \mathrm{x}_{m}\right)<\varepsilon$ for all $n, m \geq N$.

Proposition 1.5.[3] Let $(X, G)$ be a $G$ - metric space. Then the function $G(x, y, z)$ is jointly continuous in all three of its variables.

Definition 1.5.[3] A $G$ - metric space $(X, G)$ is said to be $G$-complete if every $G$-Cauchy sequence in $(X, G)$ is $G$-convergent in $X$.

Proposition 1.6.[3] A $G$ - metric space $(X, G)$ is $G-$ complete if and only if $\left(X, d_{G}\right)$ is a complete metric space.

Proposition 1.7.[3] Let $(X, G)$ be a $G$ - metric space. Then, for any $x, y, z, a$ in $X$ it follows that:

(i) If $G(x, y, z)=0$, then $x=y=z$,

(ii) $G(x, y, z) \leq G(x, x, y)+G(x, x, z)$,

(iii) $G(x, y, y) \leq 2 G(y, x, x)$,

(iv) $G(x, y, z) \leq G(x, a, z)+G(a, y, z)$,

(v) $G(x, y, z) \leq^{2} / 3(G(x, y, a)+$ $G(x, a, z)+G(a, y, z))$

(vi) $G(x, y, z) \leq(G(x, a, a)+$ $G(y, a, a)+G(z, a, a))$.

In 1996, Jungck [12] introduced the notion of weakly compatible maps as follows:

Definition 1.6.[12] A pair of self mappings $(f, g)$ of a metric space is said to be weakly compatible if they commute at the coincidence points i.e. $f u=g u$ for some $u$ in $X$, then $f g u=g f u$. 
Definition 1.7[13,14]: Two self mappings $f$ and $g$ of a Symmetric $G$-metric space $(X, G)$ are said to be occasionally weakly compatible (owc) iff there is a point $x$ in $X$ which is coincidence point of $\mathrm{f}$ and $g$ at which $f$ and $g$ commute.

Lemma 1.1[13,14]: Let $(X, G)$ be a Symmetric $G$ metric space. $f$ and $g$ be self maps on $X$ and let $f$ and $g$ have a unique point of coincidence, $w=f x=g x$, then $w$ is the unique common fixed point of $f$ and $g$.

Proof: Since $f$ and $g$ are $o w c$, there exists a point $x$ in $X$ such that $f x=g x=w$ and $f g x=g f x$. Thus, $f f x=f g x=$ $g f x$, which says that $f f x$ is also a point of coincidence of $f$ and $g$. Since the point of coincidence $w=f x$ is unique by hypothesis, $g f x=f f x=f x$, and $w=f x$ is a common fixed point of $f$ and $g$.

Moreover, if $z$ is any common fixed point of $f$ and $g$, then $z=f z=g z=w$ by the uniqueness of the point of coincidence.

In this paper, we prove new common fixed point theorem in Symmetric $G$-metric space. While proving our result, we utilize the idea of occasionally weakly compatible maps due to Al-Thagafi and N. Shahzad. Our result substantially generalize and improve a multitude of relevant common fixed point theorems of the existing literature in $G$ - metric space.

\section{Main Result}

Following to Matkowski[15], let $\Phi$ be the set of all functions $\phi$ such that $\phi:[0,+\infty) \rightarrow[0,+\infty)$ be a non-decreasing function with $\lim _{n \rightarrow+\infty} \phi^{n}(t)=0$ for all $t \in[0,+\infty)$. If $\phi \in \Phi$, then $\phi$ is called $\Phi$ - map. If $\phi$ is $\Phi$ - map, then it is an easy matter to show that

(1) $\phi(t)<t$ for all $t \in[0,+\infty)$;

(2) $\phi(0)=0$.

From now unless otherwise stated, we mean by $\phi$ the $\Phi$ - map. Now, we introduce and prove our result.
Theorem 2.1: Let $(X, G)$ be a Symmetric G-metric space. If $f$ and $g$ are owc self maps on $\mathrm{X}$ and

$G(f x, f y, f y) \leq \phi\left[\max \left\{\begin{array}{l}G(g x, g y, g y), \\ G(g x, f y, f y), \\ G(g y, f x, f x), \\ G(g y, f y, f y)\end{array}\right\}\right]$

for all $x, y$ in $X$. Then $f$ and $g$ have a unique common fixed point in $X$.

Proof: Since $f$ and $g$ are $o w c$, there exist a point $u$ in $X$ such that $f u=g u$ and $\quad f g u=g f u$. We claim that $f u$ is the unique common fixed point of $f$ and $g$. We first assert that $f u$ is a fixed point of $f$.

For, if $f f u \neq f u$, then from equation (1), we get

$\begin{aligned} G(f u, f f u, f f u) & \leq \phi\left[\max \left\{\begin{array}{l}G(g u, g f u, g f u), \\ G(g u, f f u, f f u), \\ G(g f u, f u, f u), \\ G(g f u, f f u, f f u)\end{array}\right\}\right] \\ & =\phi\left[\max \left\{\begin{array}{l}G(f u, f f u, f f u), \\ G(f u, f f u, f f u), \\ G(f f u, f u, f u), \\ G(f f u, f f u, f f u)\end{array}\right\}\right]\end{aligned}$

$$
=\phi\left[\max \left\{\begin{array}{l}
G(f u, f f u, f f u), \\
G(f u, f f u, f f u), \\
G(f f u, f u, f u), \\
0
\end{array}\right\}\right]
$$$$
=\phi\left[\max \left\{\begin{array}{l}
G(f u, f f u, f f u), \\
G(f u, f f u, f f u), \\
G(f u, f f u, f f u), \\
0
\end{array}\right\}\right]
$$$$
=\phi[G(f u, f f u, f f u)]
$$$$
<G(f u, f f u, f f u)
$$

a contradiction. So $f f u=f u$ and $f f u=f g u=g f u=f u$. Hence $f u$ is a common fixed point of $f$ and $g$.

Now we prove uniqueness. Suppose that $u, v$ in $X$ such that $f u=g u=u$ and $f v=g v=v$ and $u \neq v$. Then from equation (1), 


$$
\begin{aligned}
G(u, v, v)= & (f u, f v, f v) \\
\leq & {\left[\max \left\{\begin{array}{l}
G(g u, g v, g v), \\
G(g u, f v, f v), \\
G(g v, f u, f u), \\
G(g v, f v, f v)
\end{array}\right\}\right] } \\
= & {\left[\max \left\{\begin{array}{l}
G(u, v, v), \\
G(u, v, v), \\
G(v, u, u), \\
G(v, v, v)
\end{array}\right\}\right] } \\
= & {\left[\max \left\{\begin{array}{l}
G(u, v, v), \\
G(u, v, v), \\
G(u, v, v), \\
0
\end{array}\right\}\right] } \\
= & \phi[G(u, v, v)]<G(u, v, v)
\end{aligned}
$$

a contradiction. So $u=v$. Therefore, the common fixed point of $f$ and $g$ is unique.

Theorem 2.2: Let $(X, G)$ be a Symmetric $G$-metric space. Suppose that $f, g, S, T$ are self maps on $X$ and that the pairs $\{f, S\}$ and $\{g, T\}$ are each $o w c$. If

$$
G(f x, g y, g y)<\max \left\{\begin{array}{l}
G(S x, T y, T y), \\
G(S x, f x, f x), \\
G(T y, g y, g y), \\
G(S x, g y, g y), \\
G(T y, f x, f x)
\end{array}\right\}
$$

for all $x, y$ in $X$. Then $f, g, S$ and $T$ have a unique common fixed point in $X$.

Proof: By hypothesis, there exists points $x, y$ in $X$ such that $f x=S x$ and $g y=T y$. We claim that $f x=g y$. For, otherwise, by (2)

$$
G(f x, g y, g y)<\max \left\{\begin{array}{l}
G(S x, T y, T y), \\
G(S x, f x, f x), \\
G(T y, g y, g y), \\
G(S x, g y, g y), \\
G(T y, f x, f x)
\end{array}\right\}
$$

$$
\begin{aligned}
& =\max \left\{\begin{array}{l}
G(f x, g y, g y), \\
G(f x, f x, f x), \\
G(g y, g y, g y), \\
G(f x, g y, g y), \\
G(g y, f x, f x)
\end{array}\right\} \\
& =\max \left\{\begin{array}{l}
G(f x, g y, g y), \\
G(f x, f x, f x), \\
G(g y, g y, g y), \\
G(f x, g y, g y), \\
G(g y, f x, f x)
\end{array}\right\} \\
& =\max \left\{\begin{array}{l}
G(f x, g y, g y), \\
0,0, \\
G(f x, g y, g y), \\
G(f x, g y, g y)
\end{array}\right\} \\
& =G(f x, g y, g y)
\end{aligned}
$$

a contradiction. This implies that $f x=g y$. So $f x=S x=$ $g y=T y$. Moreover, if there is another point $z$ such that $f z=S z$, then, using (2) it follows that $f z=S z=g y=T y$ or $f x=f z$ and $w=f x=S x$ is the unique point of coincidence of $f$ and $S$. Then by lemma 1.1, it follows that $w$ is the unique common fixed point of $f$ and $S$. By symmetry, there is a unique common fixed point $z$ in $X$ such that $z=g z=T z$.

Now, we claim that $w=z$. Suppose that $w \neq z$. Using (2),

$$
\begin{aligned}
G(w, z, z)=G(f w, g z, g z) \\
<\max \left\{\begin{array}{l}
G(S w, T z, T z), \\
G(S w, f w, f w), \\
G(T z, g z, g z), \\
G(S w, g z, g z), \\
G(T z, f w, f w)
\end{array}\right\}
\end{aligned}
$$




$$
\begin{aligned}
& =\max \left\{\begin{array}{l}
G(w, z, z), \\
G(w, w, w), \\
G(z, z, z), \\
G(w, z, z), \\
G(z, w, w)
\end{array}\right\} \\
& =\max \left\{\begin{array}{l}
G(w, z, z), \\
0,0, \\
G(w, z, z), \\
G(w, z, z)
\end{array}\right\}
\end{aligned}
$$

this is a contradiction. Therefore $w=z$ and $w$ is a unique point of coincidence of $f, g, S, T$. By lemma 1.1, $w$ is the unique common fixed point of $f, g, S, T$.

Corollary 2.1: Let $(X, G)$ be a Symmetric $G$-metric space. Suppose that $f, g, S, T$ are self maps on $X$ and that the pairs $\{f, S\}$ and $\{g, T\}$ are each $o w c$.

If $G(f x, g y, g y) \leq h m(x, y, y)$

where

$$
m(x, y, y)=\max \left\{\begin{array}{l}
G(S x, T y, T y), \\
G(S x, f x, f x), \\
G(T y, g y, g y), \\
\frac{1}{2}\left(\begin{array}{l}
G(S x, g y, g y)+ \\
G(T y, f x, f x)
\end{array}\right.
\end{array}\right\}
$$

for all $x, y$ in $X$ and $0 \leq h<1$, then $f, g, S$ and $T$ have a unique common fixed point in $X$.

Proof: Since (3) is a special case of (2), the result follows immediately from Theorem 2.2.

\section{COnClusions}

In this paper, as an application of occasionally weakly compatible mappings, we prove common fixed point theorems under contractive conditions that extend the scope of the study of common fixed point theorems from the class of weakly compatible mappings to a wider class of mappings. Our result substantially generalize and improve a multitude of relevant common fixed point theorems of the existing literature in $G$ metric spaces.

\section{REFERENCES}

[1] B.C. Dhage, Generalized metric spaces and mappings with fixed point, Bull.Calcutta Math. Soc 84 (1992), 329-336.

[2] Z.Mustafa and B.Sims, Some remarks concerning D-metric spaces, Proceedings of International Conference on Fixed Point Theory and applications, Yokohama Publishers, Valencia Spain, July 1319(2004), 189-198.

[3] Z. Mustafa and B.Sims, A new approach to a generalized metric spaces, J. Nonlinear Convex Anal., 7(2006), 289-297.

[4] Z. Mustafa, H.Obiedat and F. Awawdeh, Some fixed point theorems for mappings on complete Gmetric spaces, Fixed point theorey and applications,Volume 2008, Article ID 18970, 12 pages.

[5] S. Manro, S.S. Bhatia and S. Kumar, Expansion Mapping Theorems in G - Metric Spaces, Int. J. Contemp. Math. Sciences, 5(51) (2010), 2529 2535.

[6] S. Manro, S. Kumar and S.S. Bhatia, R weakly commuting maps in $\mathrm{G}$ metric spaces, Fasciculi Mathematici, 47 (2011), 11-17.

[7] S. Manro, S. Kumar and S.S. Bhatia, Weakly Compatible maps of type (A) in $\mathrm{G}$ - metric spaces, Demonstratio Mathematica (Accepted for publication)

[8] Z.Mustafa, W. Shatanawi and M. Bataineh, Existence of fixed points results in G-metric spaces, International Journal of Mathematics and Mathematical Sciences,Volume 2009, Article ID. 283028, 10 pages.

[9] Z. Mustafa and B. Sims, Fixed point theorems for contractive mappings in complete G-metric spaces, Fixed point theory and Applications,Vol.2009, Article ID 917175, 10 Pages. 
[10]Z. Mustafa and H. Obiedat, A Fixed Points Theorem of Reich in G- metric Spaces, Cubo A Mathematics Journal. 12(1)(2010) 83-93.

[11]Z. Mustafa, F. Awawdeh and W. Shatanawi,Fixed Point Theorem for Expansive Mappings in Gmetric Spaces, Int. J. Contemp. Math. Sciences, 5(50) (2010) 2463-2472.

[12] G. Jungck, Compatible mappings and common fixed points. Int. J. Math.\& Math. Sci., 9(1986), 771-779.

[13] G.Jungck and B.E.Rhoades, Fixed point Theorems for occasionally weakly compatible mappings, Fixed point theory, 7(2006), 286-296.

[14] M.A. Al-Thagafi and N. Shahzad, Generalized Inonexpansive selfmaps and invariant approximations, Acta Math. Sinica, 24(5)(2008), 867-876.

[15] J. Matkowski, "Fixed point theorems for mappings with a contractive iterate at a point," Proceedings of the American Mathematical Society, 62(2)( 1977), 344-348.

Saurabh Manro is the Research Scholar in School of Mathematics and Computer Applications, Thapar University, Patiala (Punjab), India. He completed M.Sc (Mathematics) in year 2004 from Punjab University, Chandigarh. He is pursuing Ph.D under the kind supervision of Dr. S.S. Bhatia and Dr. Sanjay Kumar. His areas of research include Fixed point theorem in various abstract spaces like Menger spaces, Probabilistic Metric spaces, Fuzzy Metric spaces, Intuitionistic Fuzzy Metric spaces, G- Metric spaces and its applications. He has published many research papers in national / international papers till now. Some papers are ready to be published. 